Fig. 2 shows the crystal structures. In trans-(IV) nearest neighbouring anthryl groups, which are related by a centre of symmetry, interact with van der Waals contacts. Although the distance between the least-squares planes is $c a 3.4 \AA$, one anthryl group is significantly displaced with respect to the other along both the long and short axes of the anthracene, and as a result only a part of the anthracene moiety is involved in the $\pi-\pi$ interaction. The close contacts are $\mathrm{C}(1) \cdots \mathrm{C}\left(13^{\mathrm{i}}\right)=3.604(5)$, $\mathrm{C}(1) \cdots \mathrm{C}\left(14^{\mathrm{i}}\right)=3.613(5), \quad \mathrm{C}(2) \cdots \mathrm{C}\left(11^{\mathrm{i}}\right)=3.616(5)$, $\mathrm{C}(2) \cdots \mathrm{C}\left(12^{\mathrm{i}}\right)=3.384(5), \mathrm{C}(2) \cdots \mathrm{C}\left(13^{\mathrm{i}}\right)=3.227(5)$ and $\mathrm{C}(3) \cdots \mathrm{C}\left(13^{\mathrm{i}}\right)=3.663(5) \AA(\mathrm{i}=-x,-y, 2-z)$. In cis-(I), cis-(III) and cis-(IV) there are no stacking interactions observed between the anthryl groups in the nearest neighbouring molecules. The crystal structures of the four compounds explain the lack of reactivity for $[4+4]$ photodimerization of anthryl groups in the solid state.

The financial support by the Hayashi Memorial Foundation for Female Natural Scientists is acknowledged. This work was supported by a Grant-
in-Aid for Scientific Research from Ministry of Education, Science and Culture, Japan (04740294).

\section{References}

Becker, H.-D. \& Andersson, K. (1983). J. Org. Chem. 48, 4542-4549.

Becker, H.-D., Becker, H.-C., Sandros, K. \& Andersson, K. (1985). Tetrahedron Lett. 26, 1589-1592.

Becker, H.-D., Patrick, V. A. \& White, A. H. (1984). Aust. J. Chem. 37, 2215.

D'Auria, M., D’Ononfrio, F. \& VantagGi, A. (1990). Tetrahedron, 46, 7831-7840.

JOHNSON, C. K. (1965). ORTEP. Report ORNL-3794. Oak Ridge National Laboratory, Tennessee, USA.

Main, P., Hull, S. E., Lessinger, L., Germain, G., Declerce, J.-P. \& Woolfson, M. M. (1978). MULTAN78. A System of Computer Programs for the Automatic Solution of Crystal Structures from $X$-ray Diffraction Data. Univs. of York, England, and Louvain, Belgium.

Russell, A. \& Happoldt, W. B. JR (1942). J. Am. Chem. Soc. 64, 1101-1103.

Sheldrick, G. M. (1976). SHELX76. Program for Crystal Structure Determination. Univ. of Cambridge, England.

Zimmerman, H. E. \& Zuraw, M. J. (1989). J. Am. Chem. Soc. 111, 7974-7989.

Acta Cryst. (1994). B50, 112-116

\title{
On the Crystal Structures of Two Inclusion Compounds of Tris(5-acetyl-3-thienyl)methane
}

\author{
BY RICHARD E. MARSH \\ The Beckman Institute, ${ }^{*}$ California Institute of Technology, Pasadena, California 91125, USA
}

(Received 13 April 1993; accepted 31 August 1993)

\begin{abstract}
The crystal structures of the ethanol and the ethyl acetate inclusion compounds of TATM, $\mathrm{CH}\left(\mathrm{C}_{6}\right.$ $\left.\mathrm{H}_{5} \mathrm{OS}\right)_{3}$, were originally reported in space group $P 1$, with two independent TATM molecules and one solvent molecule in the unit cell. However, the pairs of TATM molecules are closely related by centers of symmetry and the structures are better described in space group $P \bar{l}$. Refinements in $P \overline{1}$ led to lower e.s.d.'s and $R$ values and to much more reasonable bond lengths, angles and $U_{i j}$ 's for the TATM molecules; however, the solvent molecules are disordered and can be described no better in $P \overline{1}$ than they were in $P 1$.
\end{abstract}

\section{Introduction}

Dillen, Roos and van Rooyen have now reported the crystal structures of four inclusion compounds of

$$
\text { * Contribution No. } 8771 \text {. }
$$

(C) 1994 International Union of Crystallography Printed in Great Britain - all rights reserved tris(5-acetyl-3-thienyl)methane, TATM, in which solvent molecules are enclathrated within a framework of TATM host molecules. In the case of the $n$-hexane solvate, the ratio of TATM to solvent is 3:1 (Roos \& Dillen, 1992); in the other three examples [benzene (van Rooyen \& Roos, 1991b), ethyl acetate (van Rooyen \& Roos, 1991a) and ethanol (Dillen \& Roos, 1992)] the ratio is $2: 1$. In the latter two cases, ethyl acetate and ethanol, the structures were described in space group P1, with two molecules of TATM and one of solvent in the unit cell. However, in both cases the two TATM molecules are closely related by a center of symmetry and refinement in $P 1$ led to improbable values for many bond lengths and angles, presumably due to the large correlations inherent in the refinement of a nearly centrosymmetric model in a noncentrosymmetric space group. Refinement in $P \overline{1}$, which requires the guest solvent molecules to be disordered, seems preferable, leading to improved $R$ values and reasonable distances and ISSN 0108-7681 (C) 1994 
Table 1. Ethyl acetate compound: coordinates, space group $P \overline{1}$

$$
\begin{gathered}
x, y, z \text { and } U_{\mathrm{cu}} \times 10^{4} . \\
U_{\mathrm{c4}}=(1 / 3) \sum_{i} \sum_{j} U_{i} a_{i}{ }^{*} a_{i}^{*} \mathbf{a}_{i} \cdot \mathbf{a}_{i} .
\end{gathered}
$$

$\begin{array}{lcccc} & x & y & z & U_{c 4} \\ \mathrm{C}(1) & 3454(3) & 3183(3) & 4061(4) & 440(8) \\ \mathrm{S}(1) & 3510(1) & 3383(1) & 9033(1) & 502(2) \\ \mathrm{C}(2) & 3525(3) & 2715(3) & 7031(5) & 478(9) \\ \mathrm{C}(3) & 3526(3) & 3515(3) & 5959(4) & 413(8) \\ \mathrm{C}(4) & 3524(3) & 4725(3) & 6779(5) & 451(8) \\ \mathrm{C}(5) & 3513(3) & 4794(3) & 8441(5) & 444(8) \\ \mathrm{C}(6) & 3482(3) & 5858(3) & 9710(5) & 522(9) \\ \mathrm{O}(1) & 3445(3) & 5725(3) & 11136(4) & 694(8) \\ \mathrm{C}(7) & 3461(5) & 7060(4) & 9142(7) & 772(13) \\ \mathrm{S}(2) & 4879(1) & 420(1) & 2408(1) & 524(2) \\ \mathrm{C}(8) & 3837(3) & 1261(3) & 2371(5) & 494(9) \\ \mathrm{C}(9) & 4158(3) & 2236(3) & 3751(4) & 406(7) \\ \mathrm{C}(10) & 5287(3) & 2286(3) & 4852(4) & 419(8) \\ \mathrm{C}(11) & 5793(3) & 1368(3) & 4307(4) & 433(8) \\ \mathrm{C}(12) & 6924(3) & 1114(3) & 5100(5) & 505(9) \\ \mathrm{O}(2) & 7259(3) & 262(3) & 4432(4) & 721(8) \\ \mathrm{C}(13) & 7662(4) & 1925(5) & 6788(6) & 752(13) \\ \mathrm{S}(3) & 207(1) & 2885(1) & 926(2) & 682(3) \\ \mathrm{C}(14) & 1646(3) & 3499(4) & 1909(5) & 569(10) \\ \mathrm{C}(15) & 2185(3) & 2800(3) & 2953(4) & 434(8) \\ \mathrm{C}(16) & 1411(3) & 1722(3) & 2954(5) & 472(8) \\ \mathrm{C}(17) & 309(3) & 1643(4) & 1916(5) & 496(9) \\ \mathrm{C}(18) & -745(3) & 681(4) & 1534(5) & 578(10) \\ \mathrm{O}(3) & -1641(3) & 768(3) & 503(4) & 903(11) \\ \mathrm{C}(19) & -688(4) & -371(4) & 2440(6) & 736(13) \\ \mathrm{C}(39)^{*} & 469(17) & 3689(33) & 6449(57) & 1924(138) \\ \mathrm{C}(40)^{*} & -153(13) & 4076(15) & 5292(19) & 943(45) \\ \mathrm{O}(7)^{*} & -1247(8) & 4011(11) & 4707(13) & 1370(35) \\ \mathrm{O}(8)^{*} & 413(9) & 5136(13) & 5027(19) & 1403(42) \\ \mathrm{C}(41)^{*} & -262(18) & 5672(17) & 2901(32) & 1369(64) \\ \mathrm{C}(42)^{*} & -452(22) & 6689(25) & 3853(28) & 1146(53) \\ & & * & & \\ & & * & & \\ & & & & \end{array}$

angles within the TATM molecules; because of the disorder, however, the solvent molecules cannot be described with confidence.

\section{TATM.EtOAc}

Triclinic; $\quad a=12.329(5), \quad b=11.229(5), \quad c=$ $8.229(5) \AA, \quad \alpha=98.42(5), \quad \beta=106.43(5), \quad \gamma=$ $99.05(5)^{\circ}, Z=1$ (van Rooyen \& Roos, $1991 a$; hereinafter, $\mathrm{RR}$ ). The $P$ l structure was refined to an $R$ value of 0.084 for 4198 reflections (RR). Many details of the resulting structure were surprising; for example, the six structurally independent but chemically equivalent $\mathrm{C}-\mathrm{C}$ bonds to the central atom $C(1)$ showed lengths ranging from $1.453(8)$ to 1.638 (6) $\AA$; there were large irregularities in the bond angles and the $U_{\text {eq }}$ values as well. After recasting the structure in $P \overline{1}$ (by moving the origin to the approximate center between the two TATM molecules and averaging the coordinates of equivalent atoms), full-matrix least-squares refinement led to an $R$ value of 0.082 for 4182 reflections recovered from SUP 54245 (16 reflections with $F_{\text {obs }}$ values of 0.0 were deleted). The total number of parameters was 281 , compared to 625 reported by RR. (The reason the ratio is not approximately $1: 2$ is because
$\mathrm{RR}$ refined the coordinates and average $B$ 's of some of the $\mathrm{H}$ atoms, whereas I placed them in fixed, calculated positions. Difference maps in the planes containing the methyl $\mathrm{H}$ atoms were ambiguous as to the orientations, so these atoms were assigned to two sets of half-populated sites. $\mathrm{H}$ atoms of the included ethyl acetate molecule were ignored.) The final $P \overline{1}$ coordinates are given in Table 1.

The new refinement has led to entirely reasonable bond lengths and angles within the host TATM molecule, equivalent values within the three 5-acetyl3-thienyl (ATM) moieties agreeing nearly as well as the e.s.d.'s would predict. The same cannot be said of the guest ethyl acetate molecule, which is perforce disordered through a center of symmetry. Because of this disorder and the overlap that results (see Fig. 1), refinement of the individual atoms, especially with anisotropic $U_{i j}$ 's, was probably inappropriate; however, convergence to a not totally unreasonable model resulted and I could find no better way to represent the solvent area. The final coordinates of the solvent atoms (Table 1) are similar to those reported by RR; the same problems of overlap in the solvent area must also have pervaded the $P 1$ refinement $(R R)$, since the structure is very nearly centrosymmetric no matter how it is described and, accordingly, large correlations could not have been avoided.

\section{TATM.EtOH}

Triclinic; $\quad a=12.488(5), \quad b=10.372(5), \quad c=$ $8.335(5) \AA, \quad \alpha=84.95(5), \quad \beta=108.74(5), \quad \gamma=$ $98.51(5)^{\circ}, Z=1$ (Dillen \& Roos, 1992; hereinafter, DR). The structure was refined in space group $P 1$ to

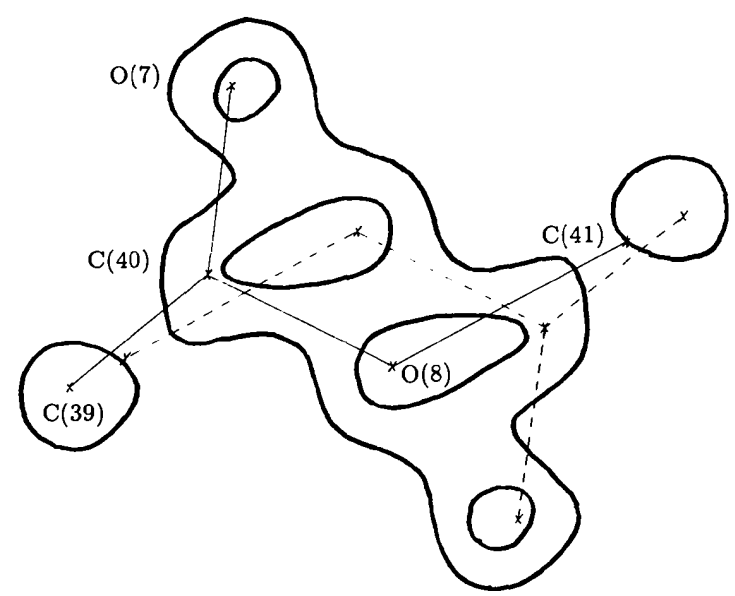

Fig. 1. Ethyl acetate complex: electron-density map through most of the major features of the solvent area. Contours are at 1.0 and $2.0 \mathrm{e} \AA^{-3}$; the center of the map is at $\left(0, \frac{1}{2}, \frac{1}{2}\right)$. The terminal methyl group $\mathrm{C}(42)$ lies about $0.8 \AA$ from this plane. The two disordered centrosymmetrically related molecules are indicated by solid and by dashed lines. 
Table 2. Ethanol compound: coordinates, space group $P \overline{1}$

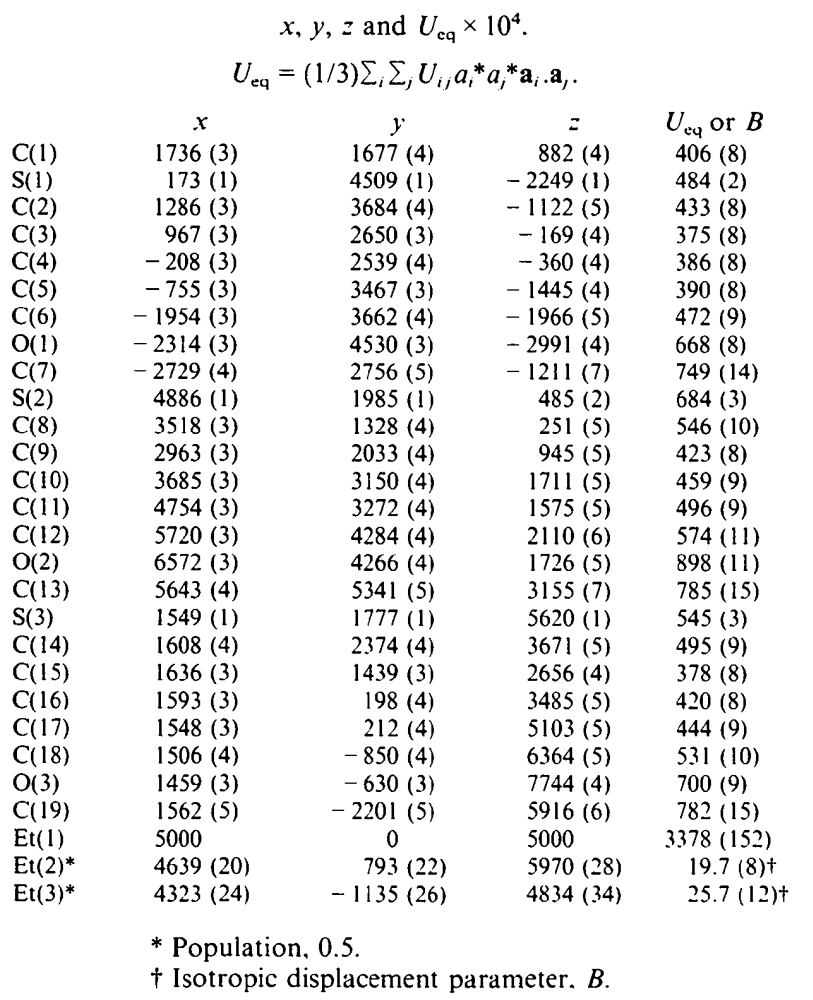

an $R$ value of 0.097 for 3786 reflections. Once again there were anomalies in the bond lengths and angles, chemically equivalent distances differing by as much as 0.26 (3) $\AA$; moreover, the $\mathrm{C}-\mathrm{C}$ and $\mathrm{C}-\mathrm{O}$ bond lengths in the enclathrated ethanol molecule needed to be constrained. Refinement in $P \overline{1}$ was based on the 3786 reflections recovered from SUP 55295. At convergence, $R$ was 0.094 for 240 parameters, compared with 483 parameters for the $P 1$ model (DR); in both cases the $\mathrm{H}$-atom positions were assumed. Once again the $P \overline{1}$ refinement led to a completely satisfactory TATM molecule but to confusion for the guest ethanol molecule. Final coordinates are in Table 2.

Attempts to model the guest ethanol molecule were frustrating and unsatisfying (as apparently had also been the case for the earlier $P 1$ model, since constraints were needed). An electron-density map through the major features in this region of the structure is shown in Fig. 2, together with the atom positions that were finally settled upon. [Note that the two maxima in Fig. 2 are not coincident with atom centers; rather, they are accounted for by overlap represented by the large $B$ 's of the three atoms and, in particular, by the very large anisotropic coefficients $U_{i j}$ of the central atom $\mathrm{Et}(1)$, which lead to an r.m.s. displacement of nearly $0.9 \AA$ in that direction.] Perhaps the only thing that can be said in favor of these positions is that they survived least-squares refinement to convergence; other arrangements of the ethanol molecule can well be imagined, but my attempts at modeling them were never satisfactory. There is clearly a guest present and studies of the very low-order reflections clearly indicated that, in this region of the structure (near $\frac{1}{2}$, $\left.0, \frac{1}{2}\right)$, about as many electrons as are present in an ethanol molecule are needed in order to match the $F_{\text {calc }}$ with the $F_{\text {obs }}$. There is no reason to doubt the presence of an enclathrated ethanol molecule, but its orientation within the cavity is unclear.

Final $P \bar{I}$ coordinates for the heavy atoms are in Table 2.*

\section{Discussion}

Unlike the earlier $P 1$ results (DR; RR), refinements of these two structures in $P \overline{1}$ have led to an entirely reasonable and apparently quite accurate description of the host TATM molecule. Formal coordinate e.s.d.'s are considerably smaller than reported earlier; more importantly, agreement among chemically equivalent bond lengths, angles and $U_{i j}$ 's is satisfactory whereas it was not before.

Quite conclusive evidence for the centrosymmetric description of the ethyl acetate compound can be found in an examination of the very weak reflections. Included in SUP 54245 are $F_{\text {obs }}$ and $F_{\text {calc }}$ values for

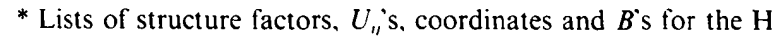
atoms, and bond lengths and angles for both compounds have been deposited with the British Library Document Supply Centre as Supplementary Publication No. SUP 71495 (28 pp.). Copies may be obtained through The Technical Editor, International Union of Crystallography. 5 Abbey Square. Chester $\mathrm{CH} 12 \mathrm{HU}$. England. [CIF reference: CR0456]

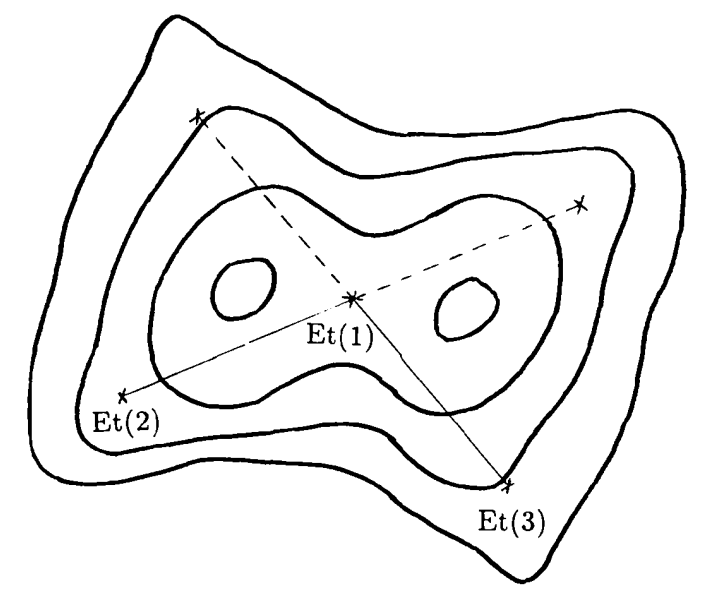

Fig. 2. Ethanol complex: clectron-density map in the plane of the major features of the solvent area. Contours are at 0.5, 1.0. 1.5 and $2.0 \mathrm{e} \AA^{3}$. The center of the map is at $\left(\begin{array}{l}1 \\ 2\end{array} 0 . \quad !\right)$. The two disordered centrosymmetrically related molecules are indicated by solid and by dashed lines. 
274 reflections with $F_{\text {obs }}=1 \mathrm{e}$ (all values were reported as integers). For these 274 reflections, the earlier $P 1$ description led to an average value of $F_{\text {calc }}$ of $1.99 \mathrm{e}$ and to an $R$ value of 1.09 ; on the other hand, the $P \overline{1}$ model derived here leads to an average $F_{\text {calc }}$ of $1.03 \mathrm{e}$ and an $R$ value of 0.59 . As has been noted many times (see, e.g., Marsh, 1981), it is these very weak reflections that are most sensitive to the centrosymmetric-noncentrosymmetric ambiguity and the convincing evidence found in this case leaves little doubt that the centrosymmetric description is preferable. The weakest reflections were not available for the ethanol compound, so a comparable test could not be done.*

The four crystallographic studies that Dillen, Roos and van Rooyen have carried out represent seven independent TATM molecules - two for the benzene adduct (van Rooyen \& Roos, 1991b), three for $n$ hexane (Roos \& Dillen, 1992) and one each for the ethanol and ethyl acetate compounds discussed here. Since each TATM molecule has three 5-acetyl-3thienyl moieties (Fig. 3), there result 21 independent determinations of the bond lengths and angles within these ATM moieties. I have calculated the weighted averages of these bond lengths and angles, the e.s.d.'s of the averages, and the goodness-of-fit values based on the assumption that all 21 measurements are equivalent. The results are summarized in Table 3 . For the bond lengths and the internal ring angles the average goodness-of-fit (GoF) is about 1.6, quite consistent with the conventional lore that formal e.s.d.'s emerging from crystallographic studies tend to be low by approximately this factor. For the

* It has been brought to my attention that Nordman \& Schmitkons (1965) have proposed a somewhat similar test for centrosymmetry: a consideration of the dependence of the ratio of observed to calculated structure amplitudes on the calculated phase angle. As the phase angle departs from 0 or $180^{\circ}$, the relative contribution of the antisymmetric component of the calculated structure factor - the $B$ term - becomes larger, as must $\left|F_{\text {calc }}\right|$ itself. This effect is most pronounced, of course, for the very weak reflections and I urge once again that all reflections be retained if there is a space-group ambiguity of this sort.

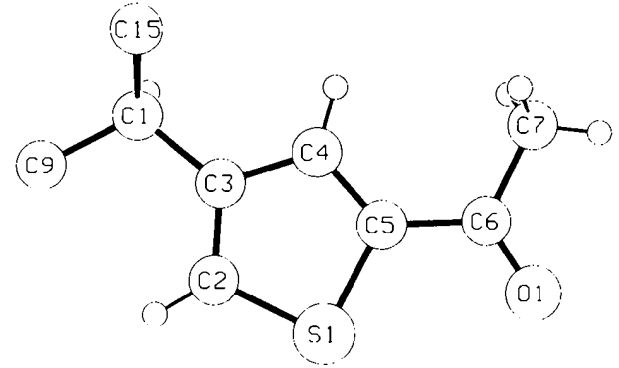

Fig. 3. A 5-acetyl-3-thienylmethyl (ATM) grouping, showing the numbering scheme used in Table 3. Atoms $C(9)$ and $C(15)$ belong to other thienyl rings and are chemically equivalent to C(3).
Table 3. Bond lengths and angles for the 5-acetyl-3thienylmethyl grouping

Values were obtained from weighted averages of 21 sets of values from four TATM crystal structures. The e.s.d.'s of the average values, in parentheses, have been multiplied by the goodness-of-fit (GoF) values, which were obtained fromi the sums of the weighted squares of deviations of the 21 individual values from their weighted average. For atom identification see Fig. 3.

$\begin{array}{lcc}\text { Bond type } & \text { Length }(\AA) & \text { GoF } \\ C(1)-C(3) & 1.517(2) & 1.3 \\ C(2)-C(3) & 1.359(2) & 1.4 \\ C(3)-C(4) & 1.417(2) & 1.4 \\ C(4)-C(5) & 1.369(2) & 1.6 \\ S(1)-C(2) & 1.707(2) & 1.6 \\ S(1)-C(5) & 1.723(2) & 2.2 \\ C(5)-C(6) & 1.466(3) & 1.9 \\ C(6)-C(7) & 1.496(2) & 1.3 \\ C(6)-O(1) & 1.216(2) & 0.9 \\ & & \\ \text { Angle type } & \text { Value ( }) & \text { GoF } \\ C(3)-C(1)-C(9) & 111.9(4) & 3.6 \\ C(1)-C(3)-C(2) & 124.9(3) & 3.0 \\ C(1)-C(3)-C(4) & 123.5(3) & 2.7 \\ C(2)-C(3)-C(4) & 111.5(2) & 1.6 \\ C(3)-C(4)-C(5) & 113.2(2) & 2.0 \\ C(3)-C(2)-S(1) & 112.8(2) & 1.6 \\ C(2)-S(1)-C(5) & 91.5(1) & 1.6 \\ S(1)-C(5)-C(4) & 111.0(2) & 2.0 \\ S(1)-C(5)-C(6) & 119.3(3) & 2.8 \\ C(4)-C(5)-C(6) & 129.7(2) & 1.8 \\ C(5)-C(6)-O(1) & 120.5(2) & 1.5 \\ C(5)-C(6)-C(7) & 118.3(2) & 2.0 \\ C(7)-C(6)-O(1) & 121.2(2) & 1.5\end{array}$

external angles at $\mathrm{C}(3)$ and $\mathrm{C}(5)$ and especially at $\mathrm{C}(1)$ the GoF's are markedly larger, clearly suggesting that the variations are real and, hence, that the molecules undergo various types of flexing as they pack together so as to form the cavities appropriate for the particular solvate molecules. Thus, the 21 measurements of these exterior angles cannot be considered as equivalent observations and there is some danger in trying to establish standard values for them. However, the bond lengths and the angles within the rings can be accepted with good confidence; one notes, for example, a significant difference between the two $\mathrm{C}-\mathrm{S}$ bond lengths.

But probably of more interest is the clathrating behavior of the TATM molecule. In forming cages for four different solvent molecules - benzene (van Rooyen \& Roos, 1991b), hexane (Roos \& Dillen, 1992), ethyl acetate (RR) and ethanol (DR) - the structure selects each time a different triclinic cell, space group $P \overline{1}$. [The axial lengths of the ethyl acetate and ethanol compounds are similar, as are the projections of the structures onto (001), but they are far from isostructural.] Thus, the compound can form at least four different types of cages, depending on the size and shape of the captured solvent. Yet these solvent molecules pack very loosely: in all four cases the $U_{\text {eq }}$ values of the solvent atoms are large $\left(0.12 \AA^{2}\right.$ or more) and in the cases of ethanol and ethyl acetate described here the noncentrosymmetric 
Table 4. Bond lengths and angles in the solvent molecules as obtained from the least-squares refinements

As a result of the disorder, these numbers are not to be trusted (see text).

\begin{tabular}{lccc}
\multicolumn{3}{c}{ Distance $(\AA)$} & Angle $\left({ }^{\circ}\right)$ \\
\multicolumn{2}{c}{ Ethyl acetate compound } & & \\
$\mathrm{C}(39)-\mathrm{C}(40)$ & 1.22 & $\mathrm{O}(7)-\mathrm{C}(40)-\mathrm{C}(39)$ & 134 \\
$\mathrm{C}(40)-\mathrm{O}(7)$ & 1.28 & $\mathrm{O}(8)-\mathrm{C}(40)-\mathrm{C}(39)$ & 111 \\
$\mathrm{C}(40)-\mathrm{O}(8)$ & 1.36 & $\mathrm{O}(8)-\mathrm{C}(40)-\mathrm{O}(7)$ & 109 \\
$\mathrm{O}(8)-\mathrm{C}(41)$ & 1.94 & $\mathrm{C}(41)-\mathrm{O}(8)-\mathrm{C}(40)$ & 118 \\
$\mathrm{C}(41)-\mathrm{C}(42)$ & 1.38 & $\mathrm{C}(42)-\mathrm{C}(41)-\mathrm{O}(8)$ & 89 \\
Ethanol compound & & & \\
$\mathrm{Et}(1)-\mathrm{Et}(2)$ & 1.41 & $\mathrm{Et}(3)-\mathrm{Et}(1)-\mathrm{Et}(2)$ & 108 \\
$\mathrm{Et}(1)-\mathrm{Et}(3)$ & 1.33 & &
\end{tabular}

guest molecule is disordered within the centrosymmetric cavity. Given the obvious ability of the TATM molecule to form cavities of several sizes and shapes, it seems surprising that the enclosed solvent does not fit in more tightly, particularly in the case of a polar solvent such as ethanol, which might be expected to form a hydrogen bond with an acetyl group of TATM. That this is not the case is clearly shown in Fig. 2: there is no concentration of electron density toward the outer region of the solvent area, where a localized $-\mathrm{OH}$ would surely lie. Nor does any acetyl $O$ atom lie within $4 \AA$ of any of the solvent atoms as finally positioned; the nearest neighbors to the solvent are a methyl group $C(7)$ of one TATM molecule and a ring atom $C(5)$ of another, at about $3.5 \AA$.

Two final comments. (1) It is doubtful - particularly in the case of the ethanol compound - that the coordinates assigned to the solvent atoms bear much resemblance to reality (see Fig. 2). It seems quite clear that these ethanol molecules occupy many different sites and are quite likely in dynamic flux at room temperature. The situation with respect to the ethyl acetate compound is somewhat clearer (Fig. 1), but by no means satisfactory. To demonstrate more clearly the situation, the bond lengths and angles calculated from the final parameters of the solvent atoms are given in Table 4; they are not dignified with e.s.d.'s (which, formally, are in the range 0.03 $0.04 \AA$ ). There were similar problems in the earlier $P 1$ refinements (DR; RR). (2) As in all cases of this type, the choice between space groups $P 1$ and $P \overline{1}$ is not a clear one, since diffraction intensities are incapable of detecting any small deviation from centrosymmetry. I can find no indication that either of these structures could be better described in $P 1$; for example, the host (TATM) atoms lying at the edges of the solvent cavities do not show the anomalous $U_{i j}$ 's that would be expected if they actually lay in two sets of positions (as would be necessary to create a noncentrosymmetric cavity). And if correct $P 1$ structures exist, it is clear, from the anomalous bond lengths, that the earlier refinements did not convi:ge to them. The advantages of the $P \overline{1}$ descriptions seem overwhelming.

\section{Computational details}

All calculations were carried out on a VAXStation 3100 under the CRYM system (Duchamp, 1964). Full-matrix least squares was based on the minimization of the quantity $\sum w\left(F_{o}^{2}-F_{c}^{2}\right)^{2}$, with weights $w$ assigned according to Hughes (1941). Final shifts were never greater than $0.05 \sigma$; excursions in final difference maps never exceeded $0.4 \mathrm{e} \AA^{-3}$. Fig. 3 was prepared with the help of ORTEPII (Johnson, 1976).

\section{References}

Dillen, J. L. M. \& Roos, H. M. (1992). Acta Cryst. C48, 2229-2231.

DuCHAMP, D. J. (1964). CRYM Crystallographic Computing System. Am. Crystallogr. Assoc. Meet., p. 29, paper B14.

Hughes, E. W. (1941). J. Am. Chem. Soc. 63, 1737-1752.

JoHnson, C. K. (1976). ORTEPII. Report ORNL-5138. Oak Ridge National Laboratory, Tennessee, USA.

MARSH, R. E. (1981). Acta Cryst. B37, 1985-1988.

Nordman, C. E. \& Schmitkons, D. L. (1965). Acta Crist. 18, 764-767.

Roos, H. M. \& Dillen, J. L. M. (1992). Acta Cryst. C48, 1882-1884.

Rooyen, P. H. van \& Roos, H. M. (1991a). Acta Cryst. C47, 2468-2470.

Rooyen, P. H. van \& Roos, H. M. (1991b). Acta Cryst. C47, 2718-2720. 